\title{
Overexpression of Yes-associated protein confers doxorubicin resistance in hepatocellullar carcinoma
}

\author{
XINYING HUO $^{1 *}$, QI ZHANG ${ }^{1 *}$, ANGELA M. LIU ${ }^{2}$, CUIJU TANG $^{1}$, YONGLING GONG $^{1}$, \\ JIANMIN BIAN ${ }^{3}$, JOHN M. LUK ${ }^{1,2}, \mathrm{ZHI} \mathrm{XU}^{1}$ and JINFEI CHEN ${ }^{1}$
}

\author{
${ }^{1}$ Department of Oncology, Nanjing First Hospital, Nanjing Medical University, Nanjing 210006, P.R. China; \\ ${ }^{2}$ Departments of Pharmacology and Surgery, National University of Singapore, 117597 Singapore, Republic of Singapore; \\ ${ }^{3}$ Department of General Surgery, Nanjing First Hospital, Nanjing Medical University, Nanjing 210006, P.R. China
}

Received October 8, 2012; Accepted November 21, 2012

DOI: $10.3892 /$ or.2012.2176

\begin{abstract}
Hepatocellular carcinoma (HCC) is one of the most aggressive malignancies worldwide and is highly resistant to chemotherapy. Yes-associated protein (YAP) is the downstream effector of the Hippo signaling pathway, which is frequently overexpressed in many types of cancers. Amplification of the YAP gene and overexpression of YAP in HCC have previously been reported to contribute to hepatocyte malignant transformation and tumor progression. In this study, we aimed to investigate the potential role of YAP in HCC chemoresistance. Overexpression of YAP resulted in resistance against doxorubicin-induced apoptosis in HCC cell lines, whereas suppression of the endogenous YAP expression by RNA interference demonstrated the reverse effect. Western blotting revealed that, following exposure to doxorubicin, YAP-overexpressing cells exhibited decreased cleaved PARP, increased phosphorylation of Akt and ERK1/2, and elevated $\mathrm{Bcl}-\mathrm{xL}$ expression in comparison to the vector control. Inhibition of YAP expression sensitized HCC cells to doxorubicin, by exhibiting increased cleaved PARP, decreased levels of phosphorylated Akt, phosphorylated ERK1/2 and Bcl-xL expression. In addition, pretreatment with the MEK1/2 inhibitor U0126 but not the PI3-K inhibitor LY294002 significantly enhanced doxorubicin-induced apoptosis and decreased $\mathrm{Bcl}-\mathrm{xL}$ expression in YAP-overexpressing HCC cells. Our data provide evidence that overexpression of YAP plays an important role in conferring doxorubicin resistance to HCC, which is at least partially mediated by YAP-induced activation of the MAP kinase pathway. Targeting YAP may be a promising adjunct for overcoming doxorubicin resistance in HCC.
\end{abstract}

Correspondence to: Dr Zhi Xu or Dr Jinfei Chen, Department of Oncology, Nanjing First Hospital, Nanjing Medical University, 68 Changle Road, Nanjing 210006, P.R. China

E-mail: michelle.xuzhi@gmail.com

E-mail: jinfeichen@sohu.com

${ }^{*}$ Contributed equally

Key words: Yes-associated protein, doxorubicin resistance, apoptosis, hepatocellular carcinoma, mitogen-activated protein kinase

\section{Introduction}

Hepatocellular carcinoma (HCC) is one of the most deadly malignancies worldwide. Surgical resection, liver transplantation, percutaneous ethanol injection (PEI) and radiofrequency ablation (RFA) may offer curative opportunities to some selected patients (1). Most HCC patients are diagnosed at an advanced stage. In this case, localregional therapies such as transarterial chemoembolization (TACE) and drug-eluting beads (DEB) are the only feasible options (2). TACE is a standard of care for patients with intermediate stage disease and includes infusing chemotherapeutics (doxorubicin, mitomycin $\mathrm{C}$ and cisplatin) mixed with lipiodol via the transarterial route into the tumor (3). With conventional chemoregimens such as doxorubicin or cisplatin, TACE has shown objective response rates of 16-61\% and prolonged patient survival (1). Nevertheless, HCC is highly resistant to chemoregimens (4) and the majority of patients die of relentless disease recurrence. To this end, optimizing drug delivery methods and enhancing drug efficacy by targeted medicine may improve clinical outcomes.

The Hippo signaling pathway, which has previously been discovered in Drosophila, plays an important role in organ-size control during embryonic development and it is conserved as a tumor-suppressor pathway in mammals (5). The core components of the Hippo pathway act in a kinase cascade, in which kinases large tumor suppressor homolog 1/2 (LATS1/2) are phosphorylated by mammalian Ste20-like kinase 1/2 (MST1/2), and this phosphorylation is coupled by salvador homolog 1 (SAV1 or WW45) (6). The cascade promotes phosphorylation and cytoplasmic retention of the Yes-associated protein (YAP, YAP1), leading to ubiquitin-mediated degradation of YAP (7) and attenuated ability to enhance transcription of genes including connective tissue growth factor (CTGF) (8) and AXL (9). YAP activation can override cell-cell contact inhibition and promote cellular growth (10), which result in malignant transformation of mammary cells $(11,12)$ and hepatocytes (9). A transgenic mouse model demonstrated that YAP overexpression causes a marked increase in liver size and eventually liver tumor formation (13). Furthermore, amplification of the YAP gene and overexpression of YAP have been found in HCC (14), lung, colon and breast cancers (15). Previously, we found that YAP overexpression is an independent prognostic 
marker associated with poor disease-free survival and overall survival in HCC (16). In this study, we further examined the role of modifying HCC cell sensitivity to doxorubicin, one of the frequently used therapeutic agents in TACE.

\section{Materials and methods}

Cell lines. The human HCC cell lines PLC/PRF/5, Hep3B and Huh7 were used as described previously (17) and maintained in DMEM (Invitrogen, Carlsbad, CA, USA) supplemented with $10 \% \mathrm{FBS}$ (Invitrogen) at $37^{\circ} \mathrm{C}$ in a humidified incubator in a $5 \% \mathrm{CO}_{2}$ atmosphere.

Ectopic expression of YAPl in Huh7 and Hep3B cells. The Huh7 cell line was transfected with the full-length YAP1 complementary DNA (NM_006106.3) plasmid (pcDNA3.1YAP1) (9) or pcDNA3.1 (Invitrogen) empty vector (control) using Lipofectamine 2000 reagent (Invitrogen), and maintained in complete medium containing $300 \mu \mathrm{g} / \mathrm{ml} \mathrm{G} 418$ (SigmaAldrich, St. Louis, MO, USA). Two separate neomycin-resistant clones (Huh7-YAP1a, Huh7-YAP1b) with high expression levels of YAP were selected for further studies. Hep3B cells were transiently transfected with the pcDNA3.1-YAP1 or pcDNA3.1 empty vector using Lipofectamine 2000. Twenty-four hours post-transfection, Hep3B-YAP1 cells were subjected to a chemosensitivity assay.

Transient knockdown of YAP1 in HCC cells. YAP1 Stealth RNAi siRNA (catalogue nos. HSS115942 and HSS115944; Invitrogen) and non-targeting siRNA (Silencer Negative control no. 1siRNA, siCon, Invitrogen) were transfected at $100 \mathrm{pmol}$ into semiconfluent PLC/PRF/5 and Huh7 cells. Cells after transfection were denoted as PLC-siYAP1a, PLC-siYAP1b, PLC-siCon, Huh7-siYAP1a, Huh7-siYAP1b and Huh7-siCon, respectively.

MTT assay. Cell viability was determined by 3-(4, 5-dimethylthiazol-2-yl)-2,5-diphenyltetrazolium bromide (MTT) assay. Cells were seeded at $1 \times 10^{3}$ cells/well onto 96-well microplates in triplicates followed by exposure to various concentrations of doxorubicin. To evaluate the involvement of the phosphatidylinositol 3-kinase (PI3-K)/Akt pathway and mitogen-activated protein (MAP) kinase pathway, Huh7-YAP1b cells were pretreated with either the PI3-K inhibitor LY294002 (25 $\mu \mathrm{M}$; Sigma-Aldrich) or the MEK1/2 inhibitor U0126 (25 $\mu \mathrm{M}$; Promega, Madison, WI, USA) dissolved in dimethyl sulphoxide (DMSO, Sigma-Aldrich) for $12 \mathrm{~h}$ before exposure to $1.5 \mu \mathrm{g} / \mathrm{ml}$ doxorubicin. At the indicated times, $20 \mu \mathrm{l}$ of MTT solution $(5 \mathrm{mg} / \mathrm{ml}$; Sigma-Aldrich) was added and incubated for $5 \mathrm{~h}$ before the end of the experiments. At the end of incubation, the culture medium was removed, and $200 \mu 1$ DMSO was added to dissolve the MTT formazan crystals at room temperature. Color development was measured at OD570 with OD655 used as the reference wavelength in a microplate reader (model 680; Bio-Rad Laboratories, Carlsbad, CA). Cell survival of untreated cells was set to $100 \%$ and survival of treated cells was expressed as a percentage relative to this value.

Western blotting. RIPA buffer containing protease inhibitor (Roche Hong Kong Ltd., Hong Kong) and phosphatase inhibitor
(Roche) was used to extract the total protein from cultured cells. For SDS-PAGE, $20 \mu \mathrm{g}$ of protein was electrophoresed onto SDS polyacrylamide gel, and then electro-transferred onto a PVDF membrane (0.4 $\mu \mathrm{m}$; Merck Millipore, Billerica, MA). After blocking, the membrane was probed with one of the following primary antibodies at a 1:1000 dilution: rabbit monoclonal antibody against human p44/42 MAP kinase, rabbit monoclonal antibody against human phospho-p44/42 MAP kinase (Thr202/ Tyr204), rabbit monoclonal antibody against Akt, rabbit monoclonal antibody against phosphor-Akt (S473), rabbit polyclonal antibody against cleaved PARP (Cell Signaling Technology Inc., Danvers, MA, USA), rabbit polyclonal antibody against human YAP (H-125), mouse monoclonal antibody against Bcl-xL, mouse monoclonal antibody against Bax (Santa Cruz Biotechnology, Inc., Santa Cruz, CA, USA). Mouse monoclonal antibody against human $\beta$-actin (Sigma-Aldrich) was use at a 1:5000 dilution as the reference control. HRP-conjugated antibodies against IgG (Invitrogen) (1:10,000 dilution) were used as the secondary antibody. Immunoreactivity signals were amplified using ECL detection reagents (GE Healthcare, Hong Kong). Bcl-xL and Bax protein expression was measured semiquantitatively using Image $\mathbf{J}$ software version 1.38 (National Institute of Health). The signal was converted to ratio by analyzing the protein band intensities of Bcl-xL relative to Bax after normalization with $\beta$-actin within the same sample.

TUNEL assay. Cell apoptosis was evaluated using ApopTag ${ }^{\circledR}$ In Situ Apoptosis Detection kits (Millipore). For induction of apoptosis, Huh7 and PLC/PRF/5 cells grown on coverslips in 6-well plates were tranfected with siRNA (scramble siRNA or siYAP1) and cultured in complete medium containing $1 \mu \mathrm{g} / \mathrm{ml}$ doxorubicin on the next day for a duration of $48 \mathrm{~h}$. Briefly, for detection of apoptosis, cells were fixed in $1 \%$ paraformaldehyde and then post-fixed in ethanol/acetic acid. After quenching endogenous peroxidase, the cells were further incubated with equilibration buffer and TdT enzyme. The reaction was halted by agitation with stop/wash buffer. After incubation with antidigoxigenin peroxidase conjugate, the cells were incubated with peroxidase substrate for color development and then counterstained with Mayer's hematoxylin. Apoptotic cells were examined and counted under the microscope at a x200 magnification from 3 random fields. The experiments were repeated twice.

Statistical analysis. The SPSS statistical package for Window version 13 (SPSS, Chicago, IL, USA) was used for data analysis. Independent Student's t-test was used to assess the effects of YAP as determined in the MTT assay, TUNEL assay and by the protein ratios of $\mathrm{Bcl}-\mathrm{xL}$ to Bax. $\mathrm{P}$-value $<0.05$ was considered to indicate a statistically significant difference.

\section{Results}

Overexpression of YAP confers resistance to doxorubicin in HCC cells. Previously, we demonstrated that YAP has a strong effect on cell growth (9). Therefore, in this study we investigated whether YAP-overexpressing HCC cells could overcome growth-inhibition when challenged with doxorubicin. Western blotting showed YAP was overexpressed in both stable transfectants (Huh7-YAP1a and Huh7-YAP1b) and 

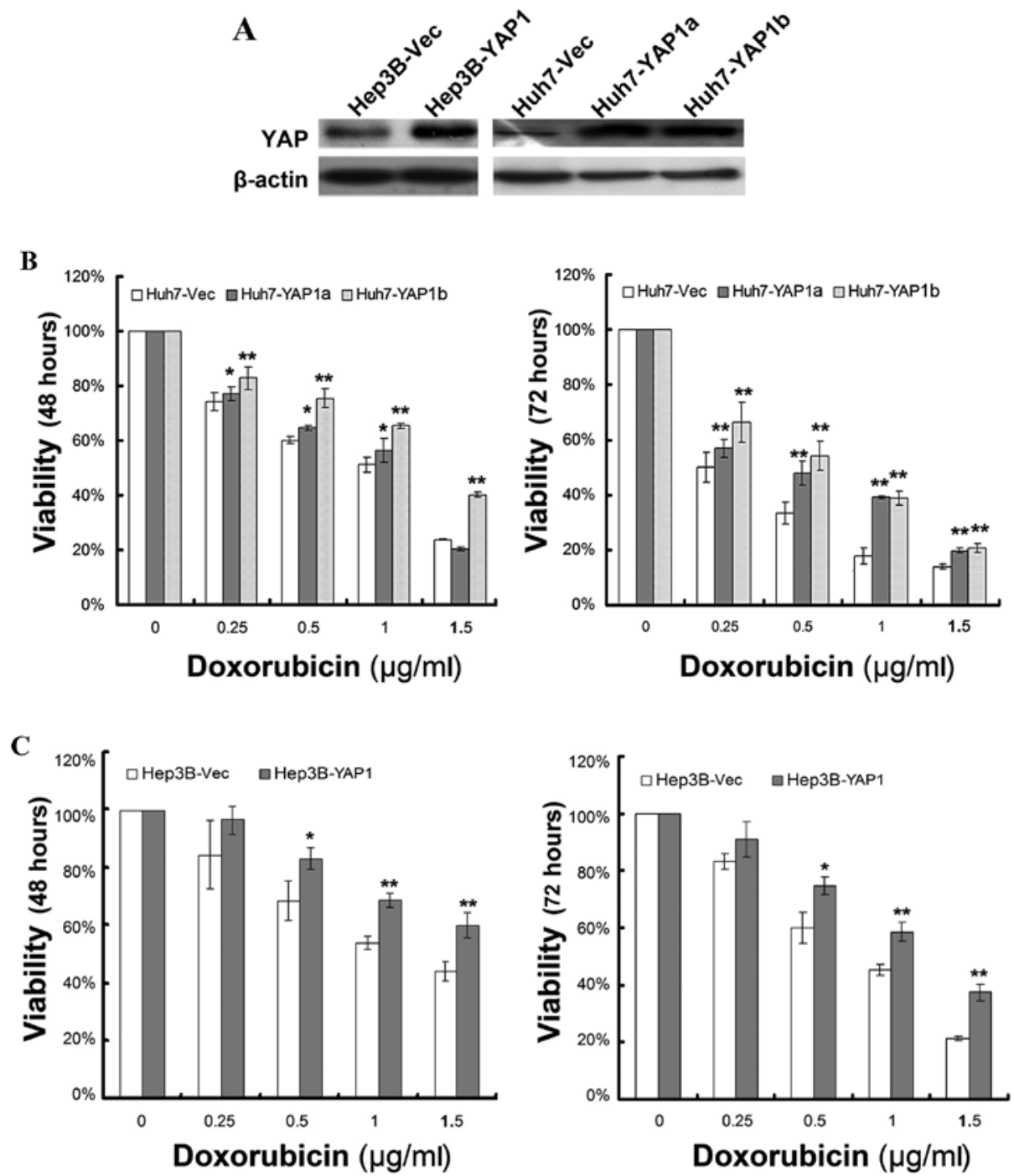

Figure 1. Overexpression of YAP confers doxorubicin-resistance to HCC cells. (A) Western blot analysis showed increased expression levels of YAP in both Huh7-YAP1 and Hep3B-YAP1 cells compared to the Huh7-Vec and Hep3B-Vec controls. (B) The viability of Huh7 cells in response to doxorubicin treatment. Huh7-YAP1 cells showed significantly enhanced cell viability when challenged with different concentrations of doxorubicin for 48 (left panel) and $72 \mathrm{~h}$ (right panel) when compared with Huh7-Vec cells. (C) The viability of Hep3B cells in response to doxorubicin treatment. Hep3B-YAP1 cells showed significantly enhanced cell viability when challenged with different concentrations of doxorubicin for 48 (left panel) and $72 \mathrm{~h}$ (right panel) when compared with Hep3B-Vec cells. Cell viability was measured by MTT assay. Comparisons were carried out by Student's t-test between the viability rates of YAP-overexpressing cells and those of control cells at the indicated concentrations of doxorubicin. Data are derived from 3 independent experiments and shown as the means \pm SD. " $\mathrm{P}<0.05$; ${ }^{* *} \mathrm{P}<0.01$.

transient transfectants (Hep3B-YAP1, Fig. 1A). Cell viability was determined by MTT assays at 48 and $72 \mathrm{~h}$ after doxorubicin treatment. As shown in Fig. 1B and C, Huh7-YAP1 and Hep3B-YAP1 cells had significantly higher viability than Huh7-Vec and Hep3B-Vec cells, respectively, at 48 and $72 \mathrm{~h}$ when treated with different concentrations of doxorubicin. The half maximal inhibitory concentrations $\left(\mathrm{IC}_{50}\right)$ at $48 \mathrm{~h}$ increased from $0.83 \mu \mathrm{g} / \mathrm{ml}$ and $1.14 \mu \mathrm{g} / \mathrm{ml}$ in Huh7-vec and Hep3B-Vec cells to $1.02 \mu \mathrm{g} / \mathrm{ml}$ and $1.73 \mu \mathrm{g} / \mathrm{ml}$, respectively, in Huh7-YAP1 and Hep3B-YAP1 cells. These data indicate that YAP decreases doxorubicin sensitivity of HCC cells.

Silencing YAP increased HCC cell sensitivity to doxorubicin. We showed that YAP overexpression desensitizes HCC cells to doxorubicin; therefore, we further explored the effects of suppressing endogenous YAP expression on doxorubicin sensitivity of HCC cells. By using RNA interference, YAP expression in Huh7 and PLC/PRF/5 cells was continuously downregulated from day 1 to day 3 post-transfection (Fig. 2A). Twenty-four hours after transient transfection with siYAPla and siYAP1b, Huh7 and PLC/PFR/5 cells were treated with various concentrations of doxorubicin for $48 \mathrm{~h}$ and the cell viability was assessed by MTT assays. As shown in Fig. 2B, Huh7 and PLC/PRF/5 cells with decreased YAP expression showed significantly lower cell viability when comparing to the scramble siRNA-transfected controls. To evaluate whether knockdown of YAP is auxiliary to doxorubicin treatment on cell apoptosis, TUNEL assay was performed. Downregulation of YAP expression significantly increased the number of apoptotic cells in the PLC/PRF/5 and Huh7 cells following treatment with doxorubicin $(1 \mu \mathrm{g} / \mathrm{ml})$ for $48 \mathrm{~h}$ (Fig. $2 \mathrm{C}$ ).

MAP kinase pathway activation is involved in YAP-mediated chemoresistance to doxorubicin. The effect of YAP on 
A
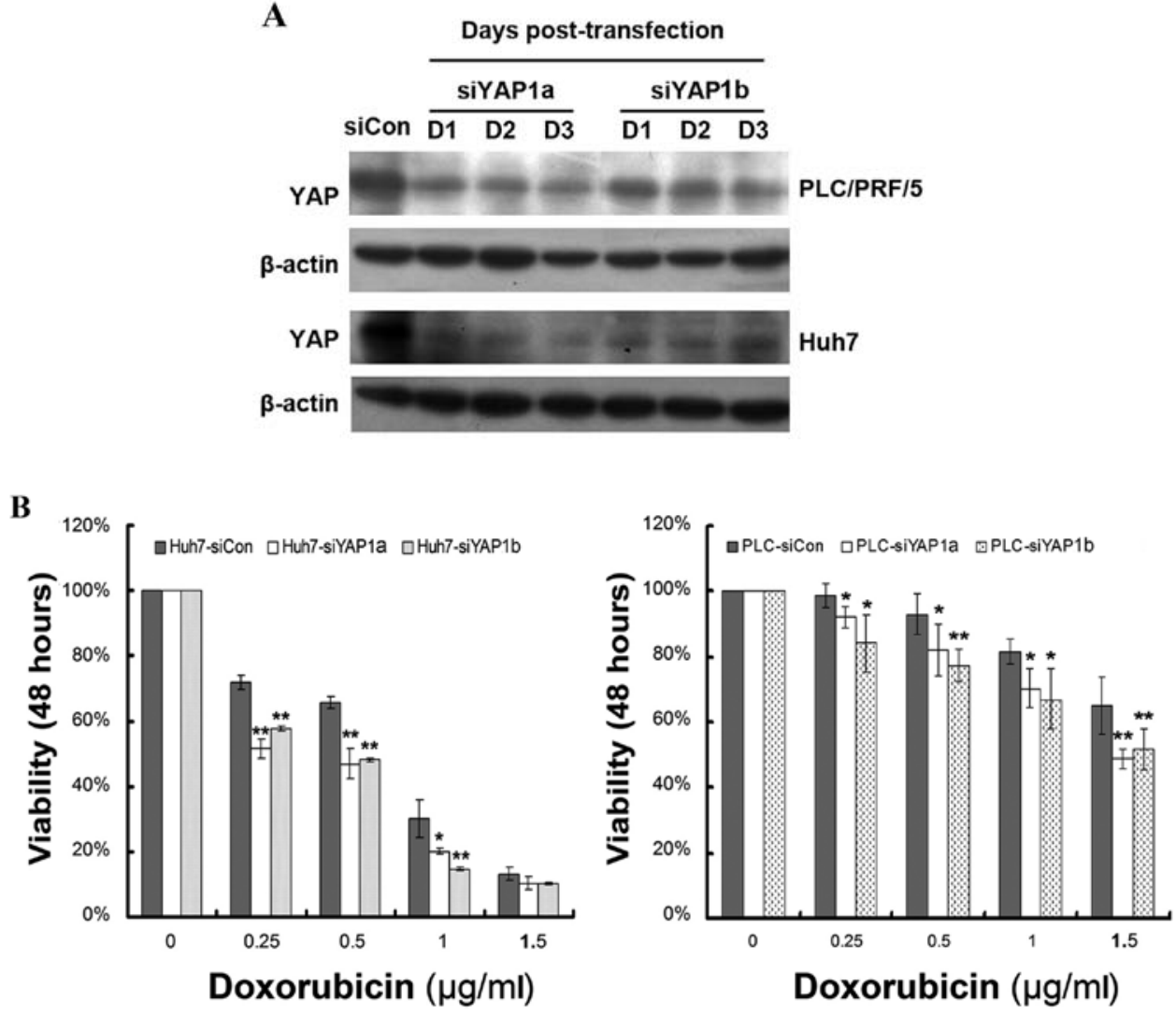

C

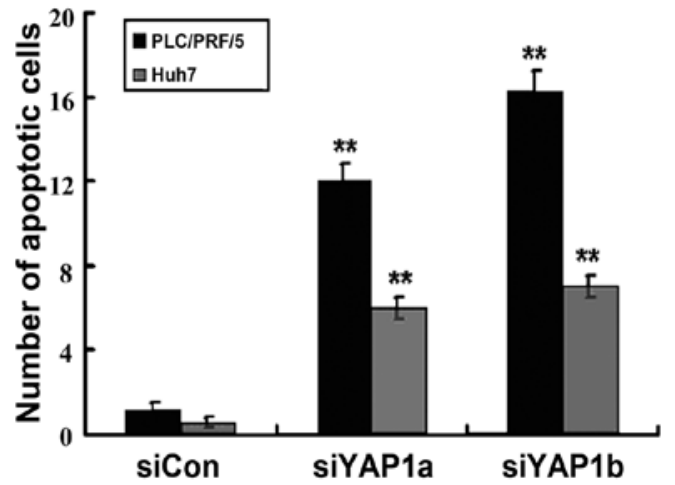

Figure 2. Suppression of endogenous YAP expression sensitizes HCC cells to doxorubicin. (A) Transient transfection of siYAP1 and siYAP2 was able to suppress YAP expression in PLC/PRF/5 and Huh7 cells continuously throughout 3 days. D1, D2 and D3 indicate day 1, 2 and 3 post-transfection. (B) siYAP1- and siYAP2-transfected Huh7 (left panel) and PLC/PRF/5 cells (right panel) showed significantly decreased cell viability when compared with their siContransfected controls when exposed to different concentrations of doxorubicin for $48 \mathrm{~h}$. Comparisons were carried out by Student's t-test between the viability rates of siYAP-transfected cells and those of the siCon-transfected cells at the same concentration of doxorubicin. Data are derived from 3 independent experiments and shown as the means $\pm \mathrm{SD}$. Comparisons were carried out by Student's $t$-test. ${ }^{*} \mathrm{P}<0.05 ;{ }^{* *} \mathrm{P}<0.01$. (C) Cell apoptosis was evaluated by TUNEL. The numbers of apoptotic cells were increased in the PLC-siYAP and Huh7-siYAP cells when compared with the number of apoptotic cells in the control cells. Data are derived from 2 independent experiments and shown as the means $\pm \mathrm{SD} .{ }^{* *} \mathrm{P}<0.01$.

doxorubicin-treated HCC cell apoptosis was further investigated by western blotting. After exposure to $1.5 \mu \mathrm{g} / \mathrm{ml}$ of doxorubicin, Huh7-YAP1b cells showed less cleaved PARP when compared to the Huh7-Vec cells (Fig. 3A) from day 1 to day 3. Meanwhile, modest upregulation of phosphorylated Akt and notably increased phosphorylated ERK1/2 were observed in the Huh7-YAP1b cells when compared to their controls (Fig. 3A), without altered total protein levels of Akt or ERK1/2. Although Bcl-xL and Bax were also induced by YAP overexpression, semi-quantitative western blotting showed that the ratios of Bcl-xL to Bax were significantly upregulated in Huh7-YAP1b cells when compared to Huh7-Vec cells after exposure to the same concentration of doxorubicin (Fig. 3B; $\mathrm{p}<0.01$ on day 2 and 3 ). In line with these results, when exposed to 1 and $1.5 \mu \mathrm{g} / \mathrm{ml}$ doxorubicin for $48 \mathrm{~h}$, PLC-siYAP1 cells showed increased cleaved PARP, modestly decreased phosphorylated Akt, notably decreased phosphorylated ERK1/2 and Bcl-xL expression when compared to PLC-siCon 
A
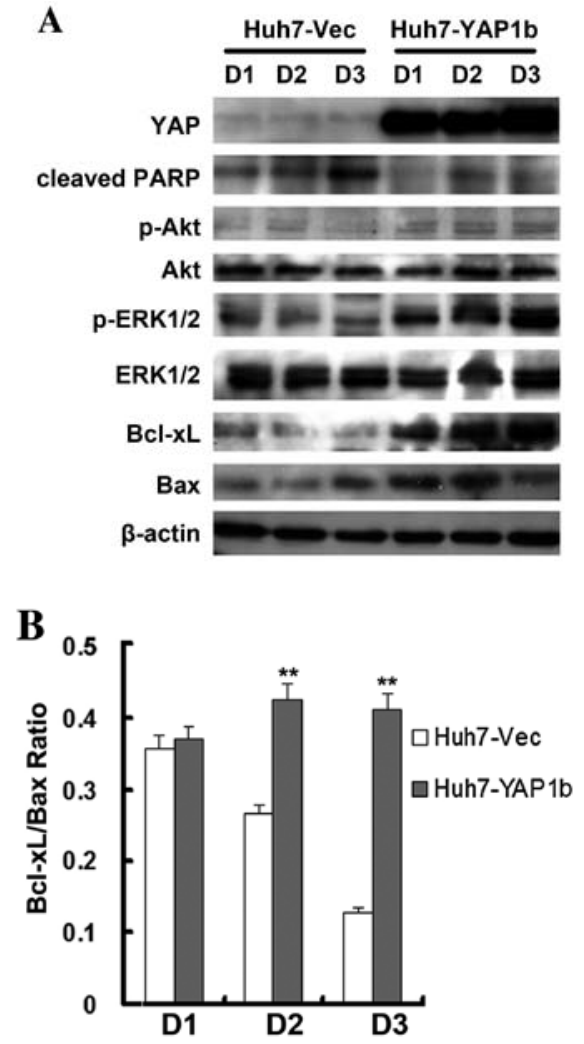

Doxorubicin $(1.5 \mu \mathrm{g} / \mathrm{ml})$

C

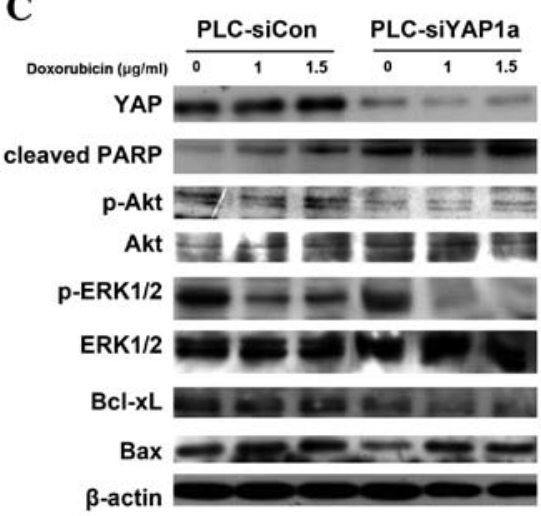

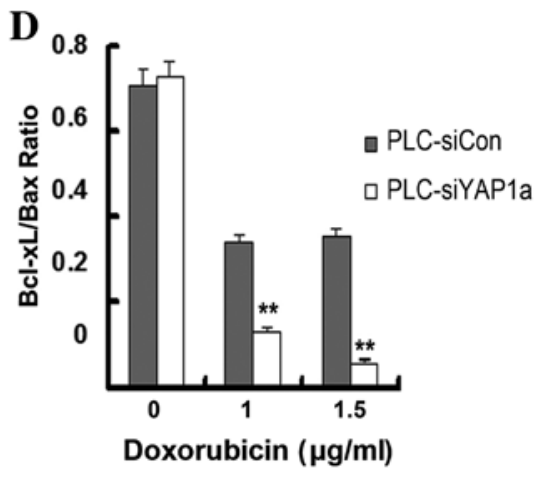

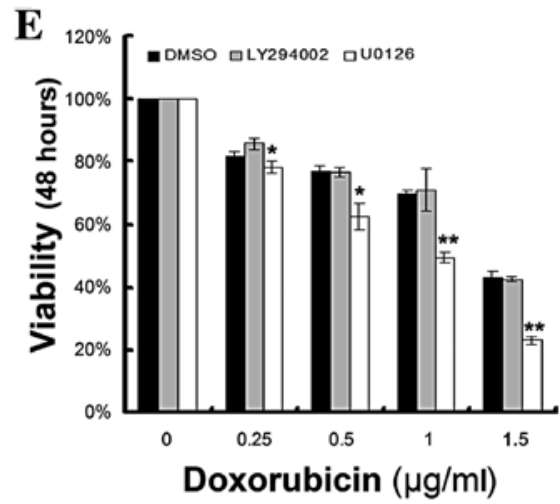

$\mathbf{F}$

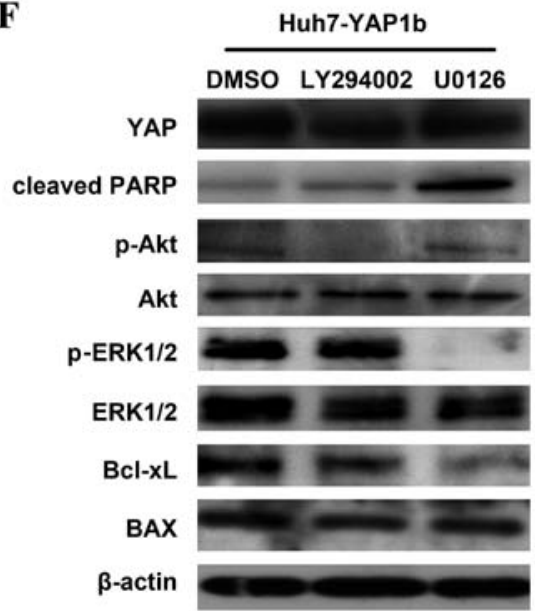

Figure 3. MAP kinase pathway mediates YAP-conferred doxorubicin resistance in HCC cells. (A) When exposed to $1.5 \mu \mathrm{g} / \mathrm{ml}$ doxorubicin, Huh7-YAP1b cells showed reduced cleaved PARP, modestly increased activated Akt, significantly upregulated phosphorylated ERK1/2 levels, elevated Bcl-xL and Bax expression compared to Huh7-Vec cells. D1, D2 and D3 indicate the doxorubicin treatment time points at day 1, 2 and 3. (B) The ratios of relative protein levels of Bcl-xL over Bax significantly increased in the Huh7-YAP1b cells when compared to the Huh7-Vec cells at day 2 and 3. Data at each time point are derived from 2 independent experiments and shown as means \pm SD. (C) When exposed to 1 or $1.5 \mu \mathrm{g} / \mathrm{ml}$ doxorubicin for $48 \mathrm{~h}$, PLC-siYAP1a cells exhibited increased cleaved PARP, modestly reduced phosphorylated Akt, significantly decreased phosphorylated ERK1/2 levels and Bcl-xL expression. (D) The ratios of the relative protein levels of Bcl-xL over Bax significantly decreased in PLC-siYAP1 than in PLC-siCon when the cells were stimulated with 1 and $1.5 \mu \mathrm{g} / \mathrm{ml}$ doxorubicin. Data at each doxorubicin concentration were derived from 2 independent experiments shown as means \pm SD. (E) Huh7-YAP1b cells were pretreated with DMSO, $25 \mu \mathrm{M} \mathrm{LY} 294002$ or $25 \mu \mathrm{M}$ U0126, followed by stimulation with doxorubicin. Cell viability was measured by MTT assay. Comparisons were carried out by Student's t-test between the viability rates of YAP-overexpressing cells and those of control cells at the indicated concentration of doxorubicin. Data are derived from 3 independent experiments and shown as means $\pm \mathrm{SD}$. ${ }^{*} \mathrm{P}<0.05 ;{ }^{* *} \mathrm{P}<0.01$. (F) When exposed to $1.5 \mu \mathrm{g} / \mathrm{ml}$ doxorubicin, only U0126pretreated Huh7-YAP1b cells showed increased cleaved PARP, significantly reduced phosphorylated ERK1/2 level and decreased Bcl-xL expression compared to the DMSO-pretreated cells. LY294002-pretreated Huh7-1b cells showed a significantly decreased phosphorylated Akt level, but not significantly altered cleaved PARP and Bcl-xL expression levels.

cells after exposure to 1 and $1.5 \mu \mathrm{g} / \mathrm{ml}$ doxorubicin for $48 \mathrm{~h}$ (Fig. 3C). Semi-quantitative analysis showed the ratios of Bcl-xL to Bax were significantly reduced in PLC-siYAP cells when compared to PLC-siCon cells (Fig. 3D, p $<0.01$ for both two dosages). These results indicate that suppression of YAP enhances doxorubicin-induced apoptosis in HCC cells.

As increased phosphorylated ERK1/2 and Akt was observed in YAP-overexpressing cells, we investigated 
whether activation of the MAP kinase or Akt signaling pathway plays roles in mediating doxorubicin resistance in YAP-overexpressing HCC cells. Pretreatment with the MAP kinase inhibitor U0126, but not the PI3-K inhibitor LY294002, significantly decreased cell viability in Huh7-YAP1b cells following exposure to doxorubicin (Fig. 3E). Moreover, when treated with both U0126 and doxorubicin, Huh7-YAP1b cells showed markedly reduced Bcl-xL expression, which was in line with the decreased phosphorylated ERK1/2 (Fig. 3F). These findings suggest that activation of the MAP kinase pathway is involved in YAP-dependent doxorubicin resistance in HCC cells.

\section{Discussion}

HCC is highly resistant to chemotherapy drugs. Currently, systemic chemotherapy fails to prolong patient survival time at large (4), and the response rate to conventional chemotherapeutics is only approximately $25 \%(18,19)$. Over the past 10 years, several targeted agents, such as cetuximab, gefitinib and sorafenib, have been evaluated in advanced HCC; nevertheless, the response rates to these drugs were only modest and their effect was to mainly promote disease stabilization (20). Recently, concurrent treatment of unresectable HCC with conventional TACE and sorafenib showed promising efficacy with manageable safety (21). Therefore, overcoming one of the major impediments of current treatment - chemoresistance, could rely on a combination of targeted therapies to improve chemocytotoxicity. In this study, we investigated the significant role of YAP in influencing doxorubicin sensitivity of HCC cells. We found that overexpression of YAP in HCC cells conferred doxorubicin resistance whereas by suppressing its endogenous expression the effect was attenuated. We suggest that this is partially attributed to YAP-induced activation of the MAP kinase pathway.

Aberrant overexpression of YAP has been described in many types of human cancers (15). The oncogenic properties of YAP include the induction of epithelial-to-mesenchymal transition (EMT), promoting resistance to apoptosis, enhancement of anchorage-independent growth capability and increasing metastatic potential (6). Previous studies have also shown that YAP is implicated in promoting chemoresistance to cisplatin in ovarian cancer $(22,23)$ and radioresistance in medulloblastoma (24). Herein, we showed that YAP expression is associated with doxorubicin sensitivity in HCC cells. Both transient and stable overexpression of YAP1 enhanced cell viability against doxorubicin in HCC cells. Compared to the controls, Huh7-YAP1 cells showed less cleaved PARP at various drug treatment time-points, indicating that YAP overexpression inhibited doxorubicin-induced apoptosis in HCC cells. In line with this, underexpression of YAP in both Huh7 and PLC/PRF/5 cells attenuated doxorubicin resistance as evidenced by the reductions in their $\mathrm{IC}_{50}$ values, increased cleaved PARP and increased numbers of apoptotic cells in the presence of doxorubicin. These results support that YAP mediates HCC cell chemoresistance to doxorubicin. Therefore, targeting YAP could be used in combination with doxorubicin to enhance chemotoxicity. Recently, dobutamine was identified as a YAP inhibitor, prohibiting YAP nuclear translocation and therefore inhibiting YAP-dependent gene transcription (25). Based on these findings, whether synergistic antitumor effects exist for HCC requires further investigation.

Our study also revealed that YAP-dependent doxorubicin resistance was strongly associated with increased expression of Bcl-xL. Both Bcl-xL and Bax belong to the Bcl-2 family and are involved in the regulation of apoptosis. Bax accelerates apoptosis by stimulating cytochrome $c$ release, which can be inhibited by Bcl-xL (26). Hence, an elevated ratio of Bcl-xL/ Bax is recognized as a phenomenon of cell fate under apoptotic damage (27). Upon apoptotic stimulation from DNA-damaging agents such as cisplatin and doxorubicin, p73-mediated Bax expression was enhanced by YAP overexpression and promoted apoptosis in breast and colon cancer cell lines $(28,29)$. Along with these findings, we found that Bax expression was upregulated in Huh7-YAP1 cells and attenuated in PLC-siYAP1 cells, which may be due to the transcriptional activation by the YAP/p73 complex. However, YAP-induced Bax expression did not increase apoptosis in the YAP-overexpressing HCC cells. Compared to the controls, enhanced cell viability and less cleaved PARP were observed at a series of doxorubicin-treated time-points in Huh7-YAP1 cells. YAP-mediated induction of Bcl-xL expression significantly elevated Bcl-xL/Bax ratios, which were associated with enhanced cell viability following DNA damage. These findings that YAP plays opposing roles in different types of cancer cells in response to DNA-damaging agents suggest that its proapoptotic or antiapoptotic activities may be cell-type dependent.

Previously, we found that YAP overexpression in HCC cells caused activation of the Akt and MAP kinase pathways (9), which are closely associated with chemoresistance (30-32). Herein, we showed that Huh7-YAP1 cells exhibited modestly phosphorylated Akt and notably phosphorylated ERK1/2 after doxorubicin treatment when compared to Huh7-Vec cells. Consistently, the levels of phosphorylated Akt, phosphorylated ERK1/2 and Bcl-xL were decreased in siYAP1-transfected cells than that in the controls. By using a pathway-specific inhibitor, we demonstrated the involvement of the MAP kinase pathway in YAP-conferred doxorubicin resistance. Only the MEK1/2 inhibitor, U0126, reduced Bcl-xL levels and sensitized Huh7YAP1b cells to doxorubicin-induced apoptosis but not the PI3-K inhibitor LY294002. The alteration of ERK1/2 phosphorylation levels was also strongly correlated with the expression levels of Bcl-xL. Inhibition of ERK1/2 activities was found to downregulate Bcl-xL expression without affecting Bax expression (33). These results suggest that YAP-conferred doxorubicin resistance to HCC cells was mediated by activation of the MAP kinase pathway and upregulation of Bcl-xL expression.

In conclusion, we provide evidence that YAP overexpression plays an important role in the chemoresistance of HCC cells to doxorubicin, which, at least partially, depends on activation of the MAP kinase pathway. Targeting YAP could sensitize cells to doxorubicin-induced cell death, suggesting that YAP may be a therapeutic target for $\mathrm{HCC}$ in combination with conventional TACE.

\section{Acknowledgements}

The study was supported by grants from the National Natural Science Foundation of China to Dr Z. Xu (grant no. 81000880) and Dr J.M. Luk (grant no. 81128080); a grant from the 
Technology Foundation for Selected Overseas Chinese Scholar, Ministry of Personnel of China to Dr Z. Xu and a grant from the Jiangsu Provincial 12th Five-Year Program on Developing Health by Technology and Education Project to Dr J. Chen.

\section{References}

1. Meza-Junco J, Montano-Loza AJ, Liu DM, et al: Locoregional radiological treatment for hepatocellular carcinoma; Which, when and how? Cancer Treat Rev 38: 54-62, 2012.

2. Huppert P: Current concepts in transarterial chemoembolization of hepatocellular carcinoma. Abdom Imaging 36: 677-683, 2011.

3. Llovet JM and Bruix J: Systematic review of randomized trials for unresectable hepatocellular carcinoma: Chemoembolization improves survival. Hepatology 37: 429-442, 2003.

4. Asghar U and Meyer T: Are there opportunities for chemotherapy in the treatment of hepatocellular cancer? J Hepatol 56: 686-695, 2012.

5. Yin M and Zhang L: Hippo signaling: a hub of growth control, tumor suppression and pluripotency maintenance. J Genet Genomics 38: 471-481, 2012.

6. Chan SW,Lim CJ, Chen L, et al: The Hippo pathway in biological control and cancer development. J Cell Physiol 226: 928-939, 2011.

7. Zhao B, Li L, Tumaneng K, Wang CY and Guan KL: A coordinated phosphorylation by Lats and CK1 regulates YAP stability through SCF(beta-TRCP). Genes Dev 24: 72-85, 2010.

8. Urtasun R, Latasa MU, Demartis MI, et al: Connective tissue growth factor autocriny in human hepatocellular carcinoma: oncogenic role and regulation by epidermal growth factor receptor/yes-associated protein-mediated activation. Hepatology 54: 2149-2158, 2012

9. Xu MZ, Chan SW, Liu AM, et al: AXL receptor kinase is a mediator of YAP-dependent oncogenic functions in hepatocellular carcinoma. Oncogene 30: 1229-1240, 2011.

10. Zhao B, Wei X, Li W, et al: Inactivation of YAP oncoprotein by the Hippo pathway is involved in cell contact inhibition and tissue growth control. Genes Dev 21: 2747-2761, 2007.

11. Overholtzer M, Zhang J, Smolen GA, et al: Transforming properties of YAP, a candidate oncogene on the chromosome 11q22 amplicon. Proc Natl Acad Sci USA 103: 12405-12410, 2006.

12. Wang X, Su L and Ou Q: Yes-associated protein promotes tumour development in luminal epithelial derived breast cancer. Eur J Cancer 48: 1227-1234, 2012.

13. Dong J, Feldmann G, Huang J, et al: Elucidation of a universal size-control mechanism in Drosophila and mammals. Cell 130: $1120-1133,2007$.

14. Zender L, Spector MS, Xue W, et al: Identification and validation of oncogenes in liver cancer using an integrative oncogenomic approach. Cell 125: 1253-1267, 2006.

15. Steinhardt AA, Gayyed MF, Klein AP, et al: Expression of Yes-associated protein in common solid tumors. Hum Pathol 39: 1582-1589, 2008.

16. Xu MZ, Yao TJ, Lee NP, et al: Yes-associated protein is an independent prognostic marker in hepatocellular carcinoma. Cancer 115: 4576-4585, 2009.
17. Liu LX, Lee NP, Chan VW, et al: Targeting cadherin-17 inactivates Wnt signaling and inhibits tumor growth in liver carcinoma. Hepatology 50: 1453-1463, 2009.

18. Ikeda M, Okusaka T, Ueno H, et al: Hepatic arterial infusion chemotherapy with epirubicin in patients with advanced hepatocellular carcinoma and portal vein tumor thrombosis. Oncology 72: 188-193, 2007.

19. Park SH, Lee Y, Han SH, et al: Systemic chemotherapy with doxorubicin, cisplatin and capecitabine for metastatic hepatocellular carcinoma. BMC Cancer 6: 3, 2006.

20. Avila MA, Berasain C, Sangro B and Prieto J: New therapies for hepatocellular carcinoma. Oncogene 25: 3866-3884, 2006.

21. Park JW, Koh YH, Kim HB, et al: Phase II study of concurrent transarterial chemoembolization and sorafenib in patients with unresectable hepatocellular carcinoma. J Hepatol 56: 1336-1342, 2012.

22. Wang P, Bai Y, Song B, et al: PP1A-mediated dephosphorylation positively regulates YAP2 activity. PLoS One 6: e24288, 2011.

23. Huang JM, Nagatomo I, Suzuki E, et al: YAP modifies cancer cell sensitivity to EGFR and survivin inhibitors and is negatively regulated by the non-receptor type protein tyrosine phosphatase 14. Oncogene: Jun. 11, 2012 (Epub ahead of print).

24. Fernandez LA, Squatrito M, Northcott P, et al: Oncogenic YAP promotes radioresistance and genomic instability in medulloblastoma through IGF2-mediated Akt activation. Oncogene 31: 1923-1937, 2012

25. Bao Y, Nakagawa K, Yang Z, et al: A cell-based assay to screen stimulators of the Hippo pathway reveals the inhibitory effect of dobutamine on the YAP-dependent gene transcription. J Biochem 150: 199-208, 2011

26. Shimizu S, Narita M and Tsujimoto Y: Bcl-2 family proteins regulate the release of apoptogenic cytochrome c by the mitochondrial channel VDAC. Nature 399: 483-487, 1999.

27. Lee TL, Yeh J, Friedman J, et al: A signal network involving coactivated NF-kappaB and STAT3 and altered p53 modulates BAX/BCL-XL expression and promotes cell survival of head and neck squamous cell carcinomas. Int J Cancer 122: 1987-1998, 2008.

28. Basu S, Totty NF, Irwin MS, Sudol M and Downward J: Akt phosphorylates the Yes-associated protein, YAP, to induce interaction with 14-3-3 and attenuation of p73-mediated apoptosis. Mol Cell 11: 11-23, 2003.

29. Lapi E, Di Agostino S, Donzelli S, et al: PML, YAP, and p73 are components of a proapoptotic autoregulatory feedback loop. Mol Cell 32: 803-814, 2008.

30. Naci D, El Azreq MA, Chetoui N, et al: $\alpha 2 \beta 1$ integrin promotes chemoresistance against doxorubicin in cancer cells through extracellular signal-regulated kinase (ERK). J Biol Chem 287: 17065-17076, 2012.

31. Yang CL, Jiang FQ, Xu F and Jiang GX: ADAM10 overexpression confers resistance to doxorubicin-induced apoptosis in hepatocellular carcinoma. Tumour Biol 33: 1535-1541, 2012.

32. Jiao $M$ and Nan KJ: Activation of PI3 kinase/Akt/HIF-1a pathway contributes to hypoxia-induced epithelial-mesenchymal transition and chemoresistance in hepatocellular carcinoma. Int J Oncol 40: 461-468, 2012

33. Boucher MJ, Morisset J, Vachon PH, Reed JC, Laine J and Rivard N: MEK/ERK signaling pathway regulates the expression of Bcl-2, Bcl-X(L), and Mcl-1 and promotes survival of human pancreatic cancer cells. J Cell Biochem 79: 355-369, 2000. 